\title{
Évaluation de la dose patient au scanner multicoupe dans un centre hospitalo-universitaire tunisien : étude de 250 patients adultes
}

\author{
S. Esseghaier ${ }^{1}$, A. Ben Mabrouk ${ }^{1 \star}$, A. Mrabet $^{2}$ et M.H. Daghfous ${ }^{1}$ \\ 1 Université de Tunis El Manar, Faculté de Médecine de Tunis, Hôpital Habib Thameur, Service de Radiologie, 1008 Montfleury, Tunisia. \\ 2 Université de Tunis El Manar, Faculté de Médecine de Tunis, 1007 Tunis, Tunisia.
}

Reçu le 14 juin 2016 - Accepté le 25 octobre 2016

\begin{abstract}
Résumé - Devant l'inflation du nombre d'examens radiologiques irradiants, particulièrement en tomodensitométrie, les pays européens, dans un souci de radioprotection, ont réagi par la transposition de la directive 97/43 de l'EURATOM complétée par la nouvelle directive 2013/59. La majorité des pays européens, dont la France, possèdent des niveaux de références diagnostiques (NRD), au-deçà desquels les valeurs dosimétriques doivent se tenir sous peine de se voir infliger des actions correctrices. En Tunisie, en l'absence de réglementation en vigueur et de NRD nationaux, nous nous sommes proposés d'évaluer notre pratique scanographique par le relevé des doses d'irradiation délivrées à des patients adultes et leurs comparaisons aux NRD français afin de juger de la nécessité d'optimisation des protocoles d'examens. L'étude, prospective et évaluative, a porté sur 250 scanners répartis en cinq types, cérébral, thoracique, abdomino-pelvien, thoraco-abdomino-pelvien et du rachis lombaire. Nous avons comparé les valeurs de $75^{\text {ème }}$ percentile des indicateurs dosimétriques (CTDI vol et DLP) par acquisition aux NRD français. L'étude a révélé un dépassement dosimétrique significatif pour nos protocoles de scanner courants et nous a incité à apporter des corrections aux protocoles utilisés et à un suivi plus rigoureux des principes de radioprotection et des règles d'optimisation et de bonne pratique en tomodensitométrie. L'évaluation dosimétrique des nouveaux protocoles est le sujet d'une étude en cours.
\end{abstract}

\begin{abstract}
Radiation dose survey for computed tomography in a Tunisian university hospital: study of 250 adult patients. Multislice computed tomography (CT) is nowadays the main source of artificial radiation for medical purposes. Diagnostic reference levels (DRLs), established by European countries for several radiological investigations, are a convenient tool to promote dose assessment. For this purpose, every department of radiology should provide an annual dosimetry report. In Tunisia, despite the lack of legal requirements, we tried to assess our CT practice by comparing the radiation doses delivered to our adult patients with French DRLs in order to make corrections to our CT protocols. We conducted a prospective and evaluative study, which included $250 \mathrm{CT}$ examinations in adult patients with standard morphologies divided into five categories: cerebral, thoracic, abdominopelvic, thoraco-abdominopelvic and lumbar spine CT. We calculated the 75th percentile values of the CTDIvol and DLP and then we compared them with correspondent DRLs. Our study showed radiation doses exceeding the French DRLs for all CT examinations. These results led to making the necessary corrections to our CT protocols and require improvement of dose optimization efforts to fulfill radiation protection principles. The dosimetric evaluation of the new protocols is the subject of an ongoing study.
\end{abstract}

Keywords: Patient, dose / dosimetry / computed tomography / optimisation

\section{Introduction}

Du fait des avantages reconnus du scanner dans l'exploration médicale, la réalisation d'examens scanographiques tous types confondus s'est accrue au cours des trois dernières décennies, représentant en France en 2012, 10,5 \% des examens radiologiques irradiants qui contribuent à 71,3\% de la dose efficace collective (IRSN, 2012).

\footnotetext{
^ asmabenmabrouk@hotmail.fr
}

Les pays européens à l'instar de la France, et afin d'être au fait des règles de bonne pratique de la radioprotection, ont adopté les recommandations de la directive européenne EURATOM 97/43 (European Atomic Energy Community) appuyées par la nouvelle directive 2013/59 (EURATOM, 1997, 2013). Ces recommandations sont réglementées en France par des textes de loi émis en 2004 et mis à jour en 2011 (JORF, 2012). Elles précisent qu'au scanner, les données dosimétriques, relatives à chaque examen et à chaque acquisition, 
doivent obligatoirement figurer dans le compte rendu; il s'agit du produit dose longueur (Dose Length Product : DLP) et de l'index de dose scanographique volumique (Volume Computed Tomography Dose Index : CTDI vol) pour les femmes jeunes (Cordoliani, 2004; JORF 2006). Elles précisent aussi que le radiologue responsable, qu'il soit dans une structure privée ou hospitalière, est tenu de procéder à une évaluation dosimétrique, annuelle et comparative aux Niveaux de Référence Diagnostiques (NRD) en vigueur, des doses délivrées aux patients (DLP et CTDI vol) lors de scanners de pratique courante. Ces NRD sont définis par acquisition pour cinq types de scanners (cérébral, thoracique, abdomino-pelvien, thoracoabdomino-pelvien et du rachis lombaire). L'évaluation dosimétrique annuelle permet de mettre à jour des dépassements dosimétriques et d'instaurer au besoin une stratégie d'optimisation des protocoles d'examens.

L'objectif de notre travail était d'évaluer notre pratique en scanner par la comparaison des doses d'irradiation délivrées à nos patients adultes, lors d'examens de pratique courante, aux valeurs françaises de NRD (JORF, 2012), en l'absence de réglementation et de NRD nationaux.

\section{Méthodes}

\subsection{Patients}

Nous avons réalisé une étude prospective et évaluative, couvrant une période de cinq mois allant de février 2015 à juin 2015 incluant 250 patients adultes des deux sexes et de morphotype standard avec un poids considéré moyen (poids maximum à $80 \mathrm{~kg}$ ) (OPRI, 2001). Les patients ont formé cinq groupes de 50 patients chacun en fonction du type de scanner réalisé.

\subsection{Scanner et protocoles}

Chaque groupe de patients a bénéficié d'un scanner parmi les cinq types, scanner cérébral, scanner thoracique, scanner abdomino-pelvien, scanner thoraco-abdomino-pelvien et scanner du rachis lombaire. Tous les examens ont été réalisés sur le même scanner multicoupe Toshiba Aquilion CXL (TokyoJapon), 64 barrettes-128 coupes mis en activité en juillet 2012. Le scanner dispose de deux options techniques de réduction de dose qui sont la collimation active et le logiciel de modulation automatique de charge et de la technique de reconstruction filtrée. Les protocoles des examens étaient standardisés, prédéfinis sur la machine afin d'uniformiser les protocoles. Le seul paramètre susceptible d'être modifié était la longueur d'acquisition adaptée à la taille du patient. Tous les scanners étaient réalisés avec une charge de $120 \mathrm{kV}$. En dehors du scanner cérébral qui était réalisé avec une charge fixée à $350 \mathrm{~mA}$, la charge était modulée automatiquement par un logiciel dédié (SURE Exposure $^{\mathrm{TM}} 3 \mathrm{D}$ Adaptive) (Kim, 2014) qui utilise la technique de modulation combinée associant une modulation dans l'axe $\mathrm{z}$ et une modulation angulaire dans l'axe $\mathrm{xy}$. La modulation automatique de la charge était ajustée à l'atténuation de la zone anatomique à explorer et au morphotype du patient en fonction d'un indice de bruit préfixé (exprimé en Déviations Standards
DS) avec une plage de milli-ampérage allant de 40 à 400 mA. Le détail des protocoles est fourni dans le Tableau 1.

Le scanner présentait en post acquisition un rapport dosimétrique sur lequel figuraient les doses délivrées exprimées en CTDI vol et en DLP pour chaque acquisition et pour la totalité de l'examen.

\subsection{Recueil et analyse des données}

Nous avons recueilli, pour tous les examens et pour une seule acquisition, les indicateurs dosimétriques, CTDI vol et DLP. Nous avons calculé les valeurs des $75^{\text {ème }}$ percentile pour chacun et nous les avons comparées aux NRD français.

\section{Résultats}

Pour le scanner cérébral, les valeurs des $75^{\text {ème }}$ percentile étaient respectivement de 94,3 mGy pour le CTDI vol et de 1801,3 mGy.cm pour le DLP dépassant les valeurs de NRD. Les indicateurs dosimétriques relevés ont dépassé les NRD dans tous les cas (Tableaux 2 et 3 et Figure 1).

Pour le scanner thoracique, les valeurs des $75^{\text {ème }}$ percentile étaient respectivement de 18,3 mGy pour le CTDI vol et de 590,6 mGy.cm pour le DLP dépassant les valeurs de NRD. Il existait un dépassement des NRD dans $52 \%$ des cas pour le CTDI vol et dans $60 \%$ pour le DLP (Tableaux 2 et 3 et Figure 2).

Pour le scanner abdomino-pelvien, les valeurs des $75^{\text {ème }}$ percentile étaient respectivement de 27,5 mGy pour le CTDI vol et de 1275,7 mGy.cm pour le DLP dépassant les valeurs de NRD. Les CTDI vol et DLP ont dépassé les NRD dans respectivement 76 et $66 \%$ des cas (Tableaux 2 et 3 et Figure 3 ).

Pour le scanner thoraco-abdomino-pelvien, les valeurs des $75^{\text {ème }}$ percentile étaient respectivement de $25,4 \mathrm{mGy}$ pour le CTDI vol et de 1218 mGy.cm pour le DLP dépassant les valeurs de NRD. Il existait un dépassement des NRD dans $80 \%$ des cas pour le CTDI vol et dans $50 \%$ pour le DLP (Tableaux 2 et 3 et Figure 4 ).

Pour le scanner du rachis lombaire, les valeurs des $75^{\text {ème }}$ percentile étaient respectivement de $46,7 \mathrm{mGy}$ pour le CTDI vol et de 1513,1 mGy.cm pour le DLP. La valeur de $75^{\text {ème }}$ percentile de CTDI vol était proche du NRD alors que celle de DLP était significativement supérieure au NRD. Les dépassements dosimétriques étaient notés dans $26 \%$ des cas pour le CTDI vol et dans $95 \%$ des cas pour le DLP (Tableaux 2 et 3 et Figure 5).

\section{Discussion}

En Tunisie, du fait de l'absence d'un cadre législatif en la matière, les NRD nationaux ne sont établis, les rapports d'irradiation évaluatifs ne sont pas réalisés dans les services de radiologie et la précision des indicateurs dosimétriques sur les comptes rendus est inconstante. De plus, la Tunisie ne dispose pas de structure de contrôle pour vérifier la conformité des doses d'irradiation délivrées aux patients aux NRD. 
Tableau 1. Paramètres d'acquisitions des protocoles standard de cinq scanners de réalisation courante. Acquisition parameters defining the standard protocols of computed tomography exams.

\begin{tabular}{|c|c|c|c|c|c|}
\hline Paramètres & Cérébral & Thoracique & Abdomino pelvien & Thoraco-abdomino pelvien & Rachis lombaire \\
\hline Tension $(\mathrm{kV})$ & 120 & 120 & 120 & 120 & 120 \\
\hline Charge (mA) & 350 & $\mathrm{R}^{*}$ & $\mathrm{R}^{*}$ & $\mathrm{R}^{*}$ & $\mathrm{R}^{*}$ \\
\hline $\begin{array}{l}\mathrm{R}^{*} \text { : Indice de bruit (DS) / charge } \\
\text { minimale - maximale (mA) }\end{array}$ & - & $10 / 40-400$ & $12,5 / 40-400$ & $10 / 40-400$ & $10 / 40-400$ \\
\hline Collimation $(\mathrm{mm})$ & $0,5 \times 32(16)$ & $0,5 \times 64(32)$ & $0,5 \times 64(32)$ & $0,5 \times 64(32)$ & $0,5 \times 64(32)$ \\
\hline $\begin{array}{l}\text { Épaisseur d'acquisition/ } \\
\text { incrément de coupe (mm) }\end{array}$ & $0,5 / 0,3$ & $05 / 0,3$ & $1 / 0,8$ & $0,8 / 1$ & $0,3 / 0,25$ \\
\hline Vitesse de rotation (seconde/rot) & 0,75 & 0,5 & 0,5 & 0,5 & 0,5 \\
\hline Durée d'acquisition (seconde) & 12,6 & 4,3 & 9,6 & 12,8 & 12,6 \\
\hline Longueur prévue (mm) & 150 & 300 & 450 & 620 & 260 \\
\hline Champ de vue (FOV) (mm) & 220,3 (Small) & 381,3 (Large) & 381,3 (Large) & 332,8 (Large) & 235,3 (Small) \\
\hline
\end{tabular}

$\mathrm{R}^{*}$ : modulation automatique de la charge

Tableau 2. Paramètres dosimétriques relevés pour les cinq types d'examen.

Dosimetric parameters for each computed tomography type.

\begin{tabular}{|c|c|c|c|c|}
\hline & Indicateurs dosimétriques & $75^{\text {ème }}$ percentile & Valeurs extrêmes & NRD* \\
\hline \multirow{2}{*}{ Encéphale } & CTDI vol (mGy) & 94,3 & $90,2-94,3$ & 65 \\
\hline & DLP (mGy.cm) & 1801,3 & $1229,6-1895,6$ & 1050 \\
\hline \multirow{2}{*}{ Thorax } & CTDI vol (mGy) & 18,3 & $6,1-18,7$ & 15 \\
\hline & DLP (mGy.cm) & 590,6 & $261,3-699,6$ & 475 \\
\hline \multirow{2}{*}{ Abdomino Pelvien } & CTDI vol (mGy) & 27,5 & $8,1-35,5$ & 17 \\
\hline & DLP (mGy.cm) & 1275,7 & $327,9-1731,1$ & 800 \\
\hline \multirow{2}{*}{ Thoraco abdomino pelvien } & CTDI vol (mGy) & 25,4 & $8,8-37$ & 20 \\
\hline & DLP (mGy.cm) & 1218 & $416,5-1576,2$ & 1000 \\
\hline \multirow{2}{*}{ Rachis lombaire } & CTDI vol (mGy) & 46,7 & $19,2-84,1$ & 45 \\
\hline & DLP (mGy.cm) & 1513,1 & $477,4-2033,9$ & 700 \\
\hline
\end{tabular}

NRD* : Niveaux de référence diagnostiques

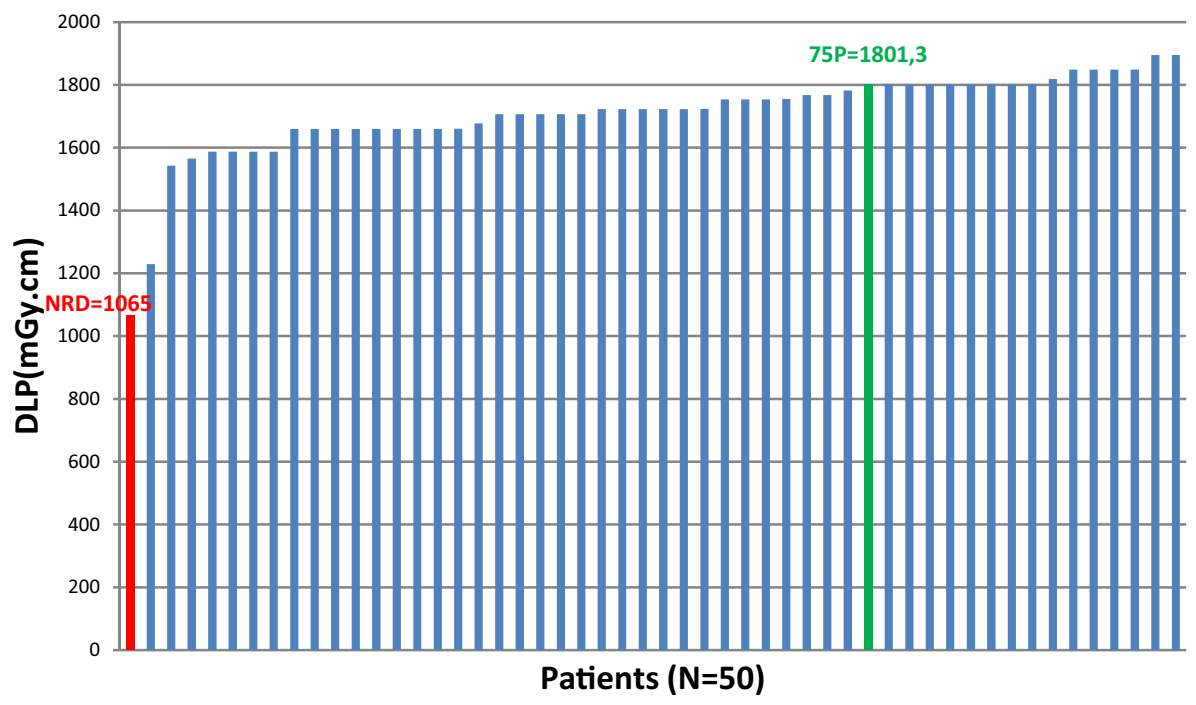

Figure 1. Distribution des valeurs de produit dose longueur (DLP) par acquisition au scanner cérébral. Distribution of the values of the DLP per acquisition for the cerebral CT scan. 


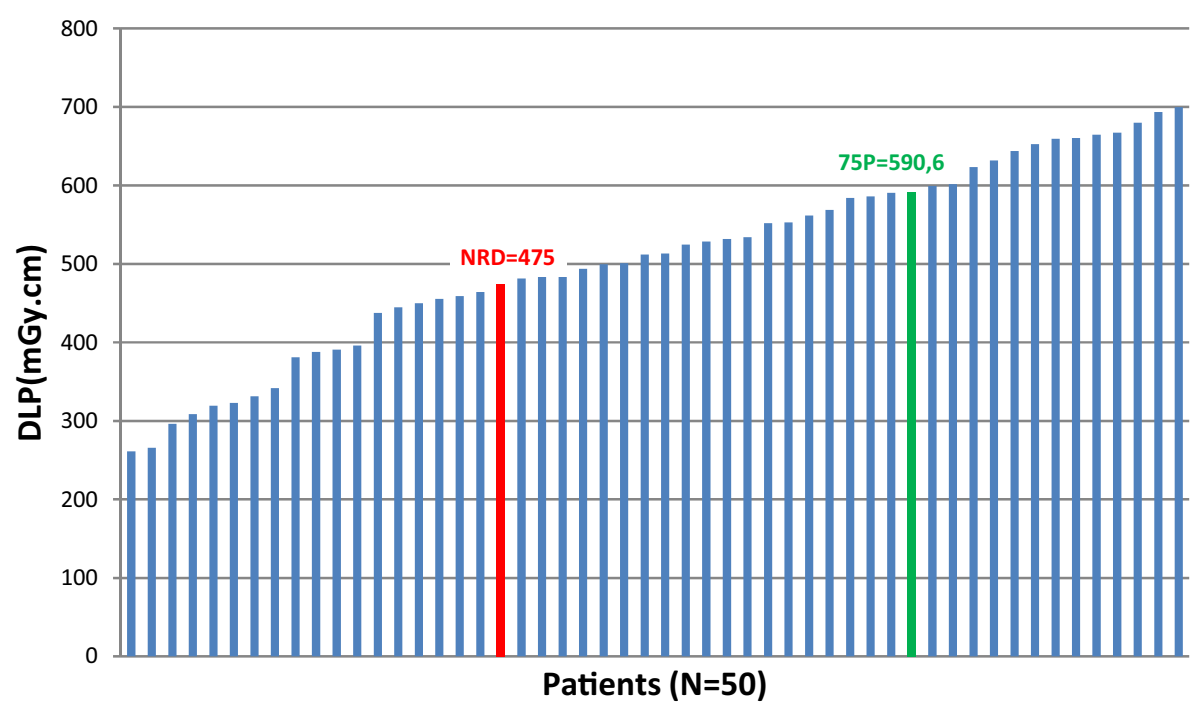

Figure 2. Distribution des valeurs de produit dose longueur (DLP) par acquisition au scanner thoracique. Distribution of the values of the DLP per acquisition for the thoracic CT scan.

Tableau 3. Distribution des valeurs de l'index de dose scanographique volumique (CTDI vol) par acquisition pour chaque type d'examen. Distribution of the values of the CTDIvol per acquisition for each CT scan type.

\begin{tabular}{|c|c|c|c|c|c|}
\hline $\begin{array}{ll}\text { CTDI vol } & \text { Protocole } \\
\end{array}$ & Cérébral & Thoracique & Abdomino-pelvien & Thoraco-abdomino-pelvien & Rachis lombaire \\
\hline Valeur minimale (mGy) & 90,2 & 6,1 & 8,1 & 8,8 & 19,2 \\
\hline Valeur maximale (mGy) & 94,3 & 18,7 & 35 & 37 & 84,1 \\
\hline Moyenne (mGy) & 93,24 & 15,28 & 23,15 & 23,39 & 39,31 \\
\hline Médiane (mGy) & 94,3 & 15,5 & 23,35 & 25,2 & 37,8 \\
\hline Ecart-type/moyenne (\%) & 2 & 21 & 30 & 23 & 33 \\
\hline $75^{\text {ème }}$ percentile (mGy) & 94,3 & 18,45 & 27,3 & 25,5 & 46,7 \\
\hline
\end{tabular}

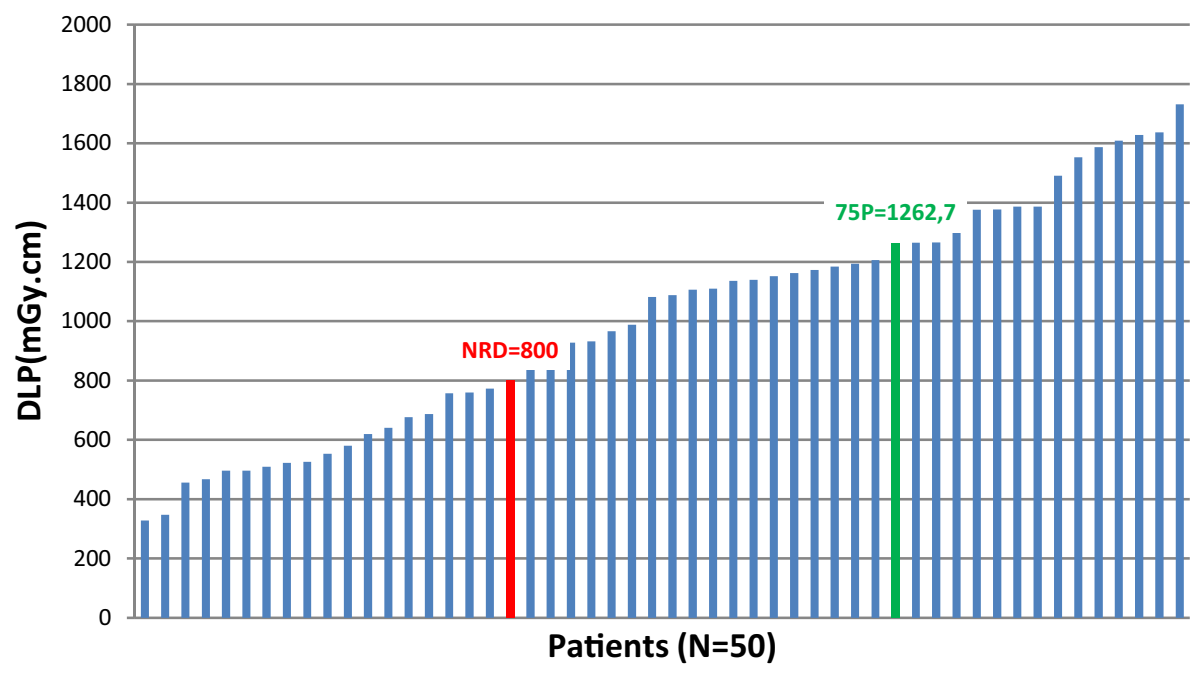

Figure 3. Distribution des valeurs de produit dose longueur (DLP) par acquisition au scanner abdomino-pelvien. Distribution of the values of the DLP per acquisition for the abdominopelvic CT scan. 


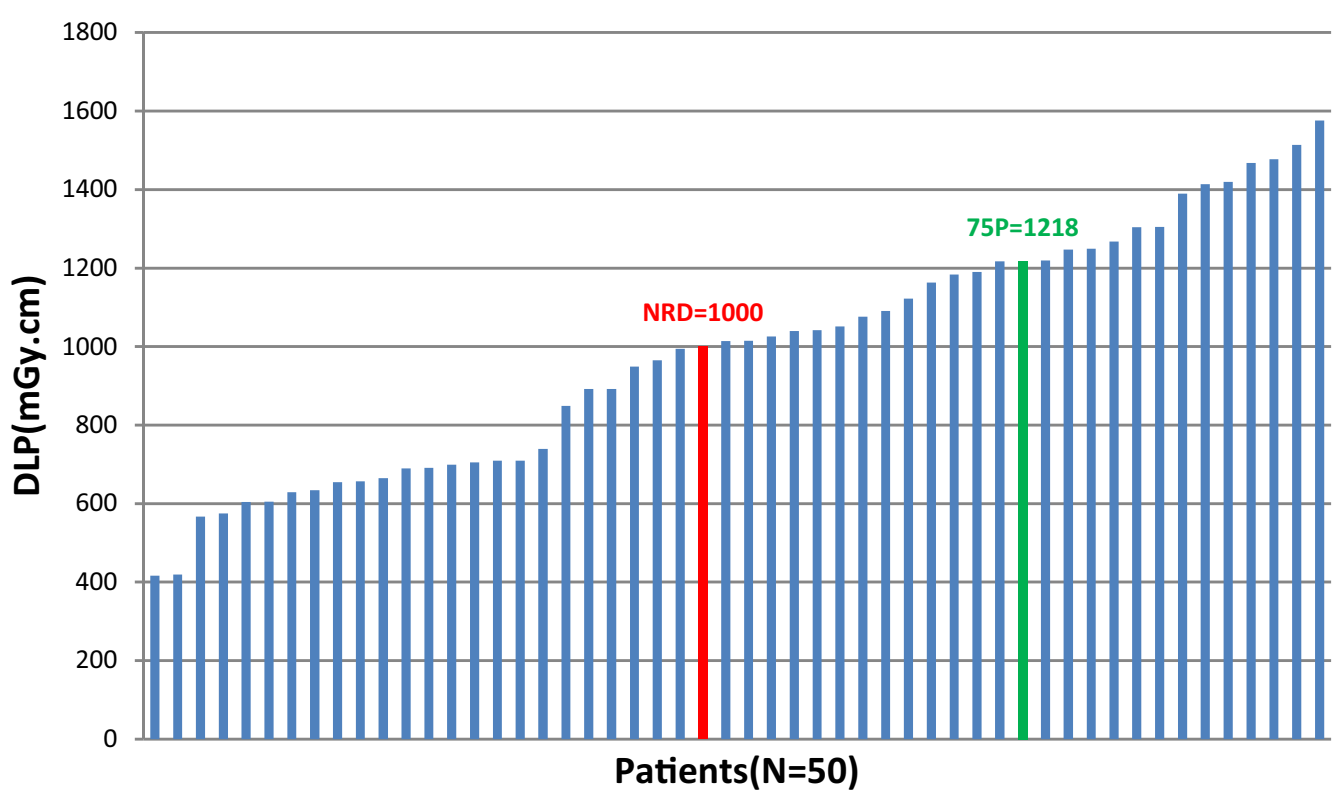

Figure 4. Distribution des valeurs de produit dose longueur (DLP) par acquisition au scanner thoraco-abdomino-pelvien. Distribution of the values of the DLP per acquisition for the thoraco-abdominopelvic CT scan.

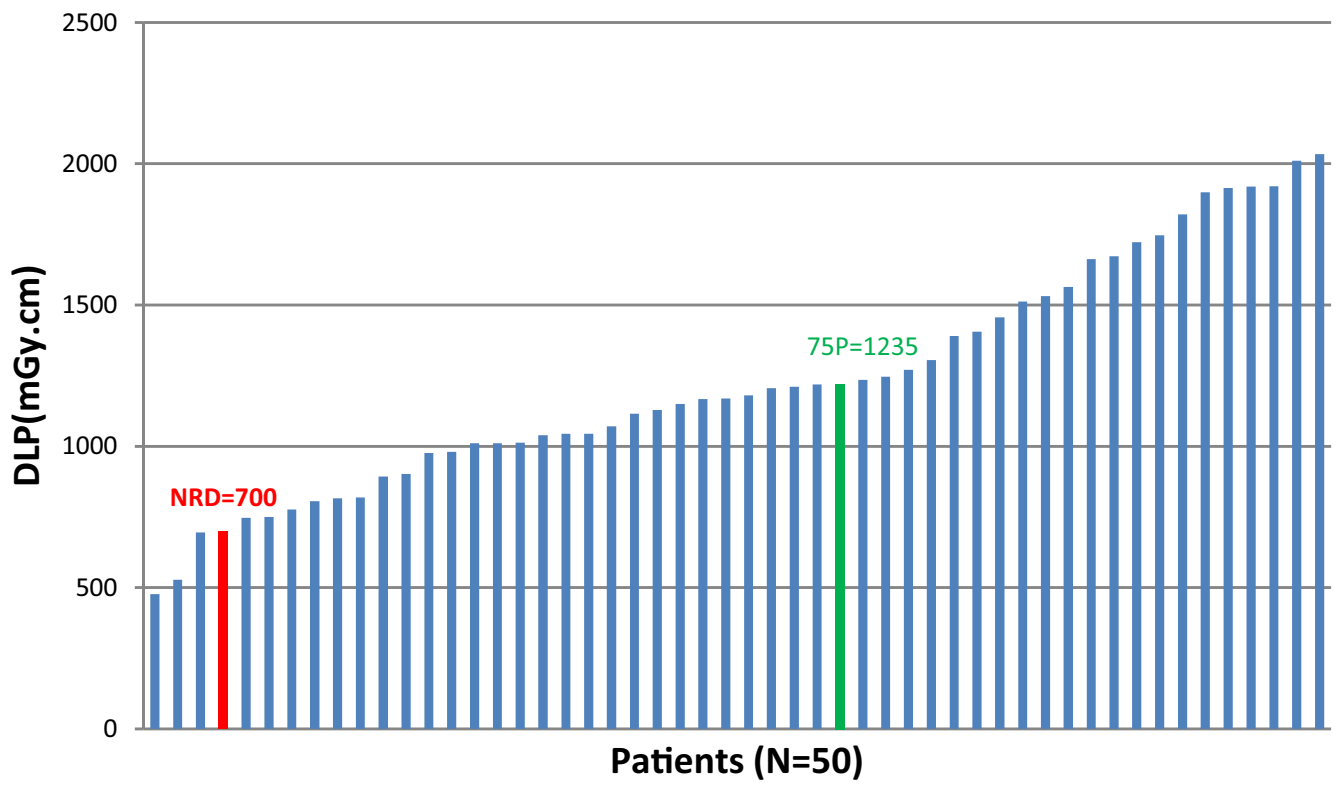

Figure 5. Distribution des valeurs de produit dose longueur (DLP) par acquisition au scanner du rachis lombaire. Distribution of the values of the DLP per acquisition for the lumbar spine CT scan.

L'ensemble engendre un manque voire une absence totale de connaissance des doses délivrées à nos patients. Le but de notre étude était de réaliser des rapports dosimétriques pour cinq types de scanners courants et nous avons comparé les valeurs des $75^{\text {ème }}$ percentile obtenues aux derniers NRD de la France, parus et réglementés par la loi de 2004 mise à jour en 2011 (JORF, 2012).

Nous avons relevé des dépassements dosimétriques pour les protocoles standards de scanners courants que nous réalisons dans notre pratique journalière et nous avons imputé ces dépassements à des paramètres techniques inadaptés sur nos protocoles.
Pour le scanner cérébral, les dépassements des NRD pouvaient être expliqués par une épaisseur d'acquisition trop fines $(32 \times 0,5 \mathrm{~mm})$ responsable d'une majoration du phénomène de pénombre (Cordoliani, 2014) et une charge élevée fixée à $350 \mathrm{~mA}$. La baisse de la charge à $200 \mathrm{~mA}$ (Chan et al., 1999) ou l'emploi d'une modulation automatique de la charge avec une valeur limite maximale de $350 \mathrm{~mA}$ (Smith et al., 2007) seraient suffisants pour obtenir une qualité d'image diagnostique. Nous avons modifié le protocole avec emploi d'une épaisseur d'acquisition millimétrique et d'une charge de $200 \mathrm{~mA}$.

Pour le scanner thoracique, le dépassement des NRD, pouvait être expliqué par une épaisseur d'acquisition fine fixée 
Tableau 4. Comparatif des paramètres d'acquisition entre anciens et nouveaux protocoles pour les scanners cérébral, thoracique et du rachis lombaire.

Comparison of acquisition parameters between the previous and new protocols for cerebral, thoracic and lumbar spine CT scans.

\begin{tabular}{|c|c|c|c|c|c|c|c|}
\hline \multirow{3}{*}{\begin{tabular}{|l} 
Type d'examen \\
Protocole
\end{tabular}} & \multicolumn{2}{|c|}{ Cérébral } & \multicolumn{3}{|c|}{ Thoracique } & \multicolumn{2}{|c|}{ Rachis lombaire } \\
\hline & $\begin{array}{l}\text { Ancien } \\
\text { protocole }\end{array}$ & $\begin{array}{l}\text { Nouveau } \\
\text { protocole }\end{array}$ & $\begin{array}{l}\text { Ancien } \\
\text { protocole }\end{array}$ & \multicolumn{2}{|c|}{$\begin{array}{l}\text { Nouveau } \\
\text { protocole }\end{array}$} & $\begin{array}{l}\text { Ancien } \\
\text { protocole }\end{array}$ & $\begin{array}{l}\text { Nouveau } \\
\text { protocole }\end{array}$ \\
\hline & & & & $\begin{array}{l}\text { Étude médiastino } \\
\text { pulmonaire usuelle }\end{array}$ & $\begin{array}{c}\text { Étude } \\
\text { parenchymateuse }\end{array}$ & & \\
\hline Tension $(\mathrm{kV})$ & 120 & 120 & 120 & $\begin{array}{l}120 \text { et } 100 \text { si suspicion } \\
\text { d'embolie pulmonaire }\end{array}$ & 120 & 120 & 120 \\
\hline Charge (mA) & 350 & 200 & modulable & modulable & égale au poids en $\mathrm{kg}$ & modulable & modulable \\
\hline $\begin{array}{l}\text { Indice de bruit (DS) /charge } \\
\text { minimale-maximale (mA) }\end{array}$ & Non & Non & $10 / 40-400$ & $10 / 40-400$ & $10 / 40-400$ & $10 / 40-400$ & $10 / 40-400$ \\
\hline Collimation (mm) & $0,5 \times 32(16)$ & $0,5 \times 32(16)$ & $0,5 \times 64(32)$ & $0,5 \times 64(32)$ & $0,5 \times 64(32)$ & $0,5 \times 64(32)$ & $0,5 \times 64(32)$ \\
\hline $\begin{array}{l}\text { Épaisseur d'acquisition/ } \\
\text { incrément de coupe (mm) }\end{array}$ & $0,5 / 0,3$ & $1 / 0,7$ & $0,5 / 0,3$ & $1 / 0,7$ & $1 / 0,7$ & $0,5 / 0,25$ & $1 / 0,3$ \\
\hline
\end{tabular}

à $0,5 \mathrm{~mm}$, requérant la délivrance par le logiciel de modulation automatique de la charge (Yu et al., 2013), d'une charge élevée pour compenser le bruit qui augmente proportionnellement à la finesse de la coupe (Cordoliani, 2005). Nous avons décidé de ne plus employer le protocole standard indifféremment pour tous les examens et de choisir entre deux protocoles en fonction de l'indication clinique. Pour l'étude médiastinopulmonaire usuelle (étude cardiaque exclue) le scanner est réalisé avec injection de produit de contraste, à $120 \mathrm{Kv}$ avec modulation automatique de la charge et une épaisseur d'acquisition millimétrique. Une réduction supplémentaire du kilovoltage à $100 \mathrm{Kv}$ est possible pour l'exploration des artères pulmonaires. En effet, en cas de suspicion d'embolie pulmonaire, il est possible, sans altérer significativement la qualité de l'examen, d'abaisser la tension et donc la dose délivrée ; l'iode majore l'atténuation du faisceau de rayons X par la plus forte probabilité d'interactions photo-électriques entre le rayon incident et les électrons de l'atome d'iode et malgré l'augmentation du bruit, la diminution du kilovoltage renforce l'atténuation des structures artérielles par rapport aux tissus environnants, produisant ainsi un rapport signal/bruit plus élevé utile pour la détection des embols artériels pulmonaires (Tack et al., 2005). Pour l'étude parenchymateuse, au cours de maladies diffuses du poumon type pneumopathies interstitielles diffuses et maladies des voies aériennes, le scanner est réalisé sans injection de PDC et en «basse dose » à $120 \mathrm{Kv}$, à charge égale au poids du patient (en kilogrammes) et avec une épaisseur d'acquisition millimétrique. En effet, l'étude du parenchyme pulmonaire peut être réalisée avec un protocole «basse dose », compte tenu du haut contraste naturel du parenchyme pulmonaire qui tolère des examens à bruit élevé (Zwirewich et al., 1991 ; Sigal-Cinqualbre et al., 2004).

Pour les protocoles standard de scanner abdomino-pelvien et thoraco-abdomino-pelvien, nous avons considéré l'épaisseur d'acquisition millimétrique inutilement fine et requérant une charge trop élevée (McCollough et al., 2009). Nous avons majoré les épaisseurs d'acquisition (sauf en cas de nécessite d'étude vasculaire) avec au besoin, réalisation de rétroreconstructions à des épaisseurs infra millimétriques.
Pour le scanner du rachis lombaire, la valeur du $75^{\text {ème }}$ percentile de CTDI vol était proche à la valeur de NRD. La valeur de $75^{\text {ème }}$ percentile de DLP était significativement supérieure à la valeur de NRD du fait de longueurs excessives des acquisitions. Nous avons insisté auprès des opérateurs sur l'importance du respect des longueurs d'acquisition car plusieurs de nos examens remontaient au niveau vertébral dorsal ou couvraient la totalité du bassin, alors que la hauteur normale de l'acquisition doit s'étendre de la douzième vertèbre dorsale aux articulations sacro-iliaques (SFR, 2011).

Les détails des nouveaux protocoles figurent dans le Tableau 4. L'évaluation dosimétrique des nouveaux protocoles est l'objet d'une étude en cours.

Par ailleurs, il serait nécessaire d'instaurer en Tunisie, une législation complète pour la radioprotection des patients afin de disposer de NRD nationaux, de structure de contrôle et de physiciens médicaux en radiologie dans les hôpitaux publics (JORF, 2011) et de mettre en place une formation continue obligatoire du personnel médical et paramédical concerné.

\section{Conclusion}

À notre connaissance, notre étude est la première qui s'est intéressée à l'évaluation des doses d'irradiation des patients en pratique scanographique diagnostique en Tunisie. Notre étude était prospective et évaluative avec un mode de recueil de données excluant les biais de sélection des patients. La limite de notre étude est qu'elle s'est limitée à des patients de poids moyen ne dépassant pas $80 \mathrm{~kg}$, la taille n'ayant pas été prise en considération et les NRD obtenus ne sont représentatifs que pour des patients de morphologie dite «standard».

Notre étude a révélé des dépassements dosimétriques, pour nos protocoles standard de scanner courants, par rapport aux NRD retenus pour la France. Nous n'avions pas relevé ces dépassements plus tôt car, en l'absence d'obligation règlementaire, nous ne réalisions pas de contrôles évaluatifs périodiques des doses d'irradiation délivrées à nos patients. Nos résultats 
nous ont emmenés à apporter des modifications à nos protocoles qui sont le sujet d'une nouvelle évaluation dosimétrique.

\section{Références}

Chan C.Y., Wong Y.C., Chau L.F., Yu S.K., Lau P.C. (1999) Radiation dose reduction in paediatric cranial CT, Pediatr. Radiol. 29, 770775 .

Cordoliani Y.S. (2004) Doses délivrées au patient en scanographie (scanner), J. Radiol. 44, 139-145.

Cordoliani Y.S. (2005) RSNA 2004 : Quid de la radioprotection? J. Radiol. 86, 890-891.

Cordoliani Y.S. (2014) Radioprotection en milieu médical. Principes et mise en pratique. Elsevier Masson, Paris.

EURATOM (1997). Directive 97/43 du conseil du 30 juin 1997relative à la protection sanitaire des personnes contre les dangers des rayonnements ionisants lors d'expositions à des fins médicales. Paris, France.

EURATOM (2013) Directive 2013/59 du conseil du 5 décembre 2013 fixant les normes de base relatives à la protection sanitaire contre les dangers résultant de l'exposition aux rayonnements ionisants. Paris, France.

IRSN (2012) Rapport PRP-HOM N² 2014-6 Exposition de la population française aux rayonnements ionisants liée aux actes de diagnostic médical en 2012. Paris, France.

JORF (2006) Arrêté du 22 septembre 2006 relatif aux informations dosimétriques devant figurer dans un compte rendu d'acte utilisant les rayonnements ionisants. Paris, France.
JORF (2011) Décision du 11 mars 2011 modifiant la décision du 22 novembre 2007 fixant les modalités du contrôle de qualité des scanographes. Paris, France.

JORF (2012) Arrêté du 24 octobre 2011 relatif aux niveaux de référence diagnostiques en radiologie et en médecine nucléaire. Paris, France.

Kim M et al. (2014) Adaptive Iterative Dose Reduction Algorithm in CT : Effect on Image Quality Compared with Filtered Back Projection in Body Phantoms of Different Sizes, Korean J. Radiol. 15(2), 195-204.

McCollough C.H., Primak A.N., Braun N., Kofler J., Yu L., Christner J. (2009) Strategies for reducing radiation dose in CT, Radiol. Clin. North Am. 47, 27-40.

OPRI (2001) Transposition de la Directive 97/43 Euratom : Mission sur les procédures et les niveaux de référence des examens médicaux utilisant les rayonnements ionisants. Paris, France.

SFR (2011) Guide du bon usage des examens d'imagerie médicale. Paris, France.

Sigal-Cinqualbre A.B., Hennequin R., Abada H.T., Chen X., Paul J.F. (2004) Low kilovoltage multi-detector row chest CT in adults : feasibility and effect on image quality and iodine dose, Radiology 231, 169-174.

Tack D., Sourtzis S., Delpierre I., de Maertelaer V., Gevenois P.A. (2003) Low-dose unenhanced multidetector CT of patients with suspected renal colic, Am. J. Roentgenol. 180, 305-311.

Yu L. et al. (2013) Automatic selection of tube potential for radiation dose reduction in vascular and contrast-enhanced abdominopelvic CT, Am. J. Roentgenol. 201, 297-306.

Zwirewich C.V., Mayo J.R., Muller N.L. (1991) Low-dose highresolution CT of lung parenchyma, Radiology 180, 413-417.

Cite this article as: S. Esseghaier, A. Ben Mabrouk, A. Mrabet, M.H. Daghfous. Évaluation de la dose patient au scanner multicoupe dans un centre hospitalo-universitaire tunisien : étude de 250 patients adultes. Radioprotection 51(4), 271-277 (2016). 